\title{
A joint Precoding scheme and Selective Transmit Diversity
}

\author{
Mário Marques da Silva ${ }^{1}$, Américo Correia ${ }^{2}$ \\ Institute for Telecommunications ${ }^{1}, \mathrm{ADETTI}^{2}$ \\ IST, Torre Norte 11.10, Av. Rovisco Pais, 1049-001 Lisboa, Portuga 1 \\ http://www.it.pt \\ marques.silva@engenheiros.pt, $\underline{\text { americo.correia@iscte.pt }}$
}

\begin{abstract}
We propose the use of the precoding (PC) scheme, which can be used to combat both the Multiple Access Interference (MAI) and Inter-Path Interference (IPI), combined with the Selective Transmit Diversity (STD) for high data rate transmissions. The proposed scheme is considered over frequency slective Rayleigh fading channels jointly with a RAKE in the receiver. With the $\mathrm{PC}$, the increase in performance is achieved with a small increase in power processing in the BS, avoiding any need to increase complexity in the Mobile Station (MS) $[1,2,3]$. It is shown that the use of the proposed PC scheme, alone or combined with the STD achieves a performance improvement over the corresponding schemes without PC.
\end{abstract}

\section{Introduction}

Much research has been undertaken in the area of the Multi-User Detectors [4] for Direct Sequence Code Division Multiple Access (DS-CDMA) technology. It can be mainly used by Base Stations (BS) where there is enough power processing capability and where it is easier to know/estimate the uplink Channel Impulse Responses (CIR) and the spreading sequences of interfering users. However, the trend of the telecommunications is to become more and more asynchronous and with higher data rates in the downlink than in the uplink. This is useful for services like Web browsing, Data Base Access, Multimedia, etc. To obtain this, it is important to transfer the complexity as much as possible to the BS, where the power processing and electrical power available is higher, so as to combat the several sources of interference.

With the aid of pre-distorting the signals to be transmitted by the BS, the orthogonality between the signals seen by the different users can be improved. The proposed PC scheme is done taking into account the spreading sequences, transmitted symbols and the CIR of the several users present in the cell. The current approach considers a RAKE receiver in the MS. Moreover, we consider a combination of PC with the STD.

This paper is structured as follows: section 2 presents the system model for the proposed Precoding; section 3 presents the system model for the Selective Transmit Diversity; section 4 presents the performance results and analysis for the proposed schemes; the key findings of this paper are then summarized in section 5 . 
2 Mário Marques da Silva1, Américo Correia2

\section{System Model for the Precoding}

Multi-user detection has been investigated mostly from the viewpoint of receiver optimization. However, in the downlink situation, the MS usually does not have access to information needed to perform the Multi-user Detection (MUD). Such information consists of sprea ding sequences, CIR and the symbols transmitted by different users. Additionally, the processing capability and power energy at the MS is limited. For these reasons, we propose to transfer the complexity from the MS to the BS, and hence, we implement the PC scheme. The transmitted signal is $x(t)=s^{T}(t) \mathbf{T A b}$, where $\mathbf{T}$ is a $(2 M+1) K \mathrm{X}(2 M+1) K$ matrix to be chosen according to some optimality criterion. Therefore, with PC, the vector of MF output is given by $\mathbf{y}=\mathbf{R T A b}+\mathbf{z}$.

Thus, the optimum PC transformation $\mathbf{T}$, which minimizes the bit error rate (BER), is derived in [1] as $\mathbf{T}=\mathbf{R}^{-1}$. This makes $\mathbf{y}=\mathbf{A} \mathbf{b}+\mathbf{z}$.

Assuming that the signal of each user is subject to frequency selective multipath fading, the received signal at the $p^{\text {th }}$ receiver can be expressed as [1]:

$$
\begin{gathered}
r_{p}(t)=S_{p}(t, b)+n_{p}(t) \\
S_{p}(t, b)=\sum_{i=-M}^{M} \sum_{k=1}^{K} \sum_{l=1}^{L-1} b_{k}(i) A_{k}(i) \alpha_{p}^{l}(i) s_{k}\left(t-i T_{b}-l T_{c}\right)
\end{gathered}
$$

being $T_{C}$ the chip duration, $b_{k}(i)$ the transmitted symbol of the $k^{\text {th }}$ user, $k=1, \ldots, K$, $i=-M, \ldots, M, A_{k}(i)$ is the amplitude of the $k^{\text {th }}$ signal in the $i^{\text {th }}$ symbol interval. $\alpha_{p}^{l}(i)$ represents the gain of the channel of the $t^{h}$ multipath component during the $t^{t h}$ symbol interval over the channel between the transmitter and the $p^{\text {th }}$ receiver. When we consider $(L-1) T_{c}<T_{b}$ the window should have size 3 , i.e., $M=[-1,0,1]$. Thus, the output of the RAKE receiver comes:

$$
y_{p}(i)=\sum_{n=0}^{L-1} \int_{i T_{b}+n T_{c}}^{(i+1) T_{b}+n T_{c}} \alpha_{p}^{n}(i) r_{p}(t) s_{p}\left(t-i T_{b}-n T_{c}\right) d t
$$

To make this notation compact, we define a $(2 M+1) K \times(2 M+1) K \quad \mathbf{R}(l-n)$ matrix as in [1]:
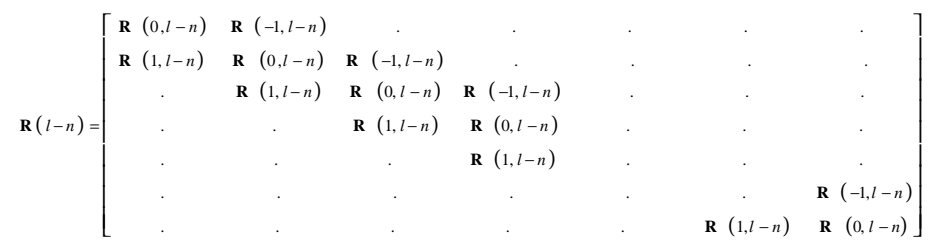

where, due to the size window $3,\{\mathbf{R}(l-n)\}_{i, j}=0$ for every $i, j$, such that $|i-j|>1$. Additionally, we define $\mathbf{R}(m, l-n)$ as a $K \times K$ matrix whose values are: 


$$
R_{p, k}(m, l) \int_{-\infty}^{+\infty} s_{p}(t) s_{k}\left(t-m T_{b}-l T_{c}\right) d t
$$

Assuming that multipath intensity profiles do not change over the block length, we define [1]:

$$
\begin{aligned}
& \mathbf{A}=\operatorname{diag}\left[A_{1}(-M), \ldots, A_{K}(-M), \ldots, A_{1}(M), \ldots, A_{K}(M)\right]_{(2 M+1) K(2 M+) K} \\
& \alpha(l)=\operatorname{diag}\left[\alpha_{1}^{l}(-M), \ldots, \alpha_{K}^{l}(-M), \ldots, \alpha_{1}^{l}(M), \ldots, \alpha_{K}^{l}(M)\right]
\end{aligned}
$$

and $K(2 M+1) \times 1$ vectors such that:

$$
\begin{aligned}
& \mathbf{b}=\left[b_{1}(-M)^{T}, \ldots, b_{K}(-M)^{T}, \ldots, b_{1}(M)^{T}, \ldots, b_{K}(M)^{T}\right]^{T} \\
& \mathbf{z}=\left[z_{1}(-M)^{T}, \ldots, z_{K}(-M)^{T}, \ldots, z_{1}(M)^{T}, \ldots, z_{K}(M)^{T}\right]^{T}
\end{aligned}
$$

Hence, we express the vector of the RAKE receiver outputs at all $K$ sites as:

$$
\begin{aligned}
\hat{\mathbf{y}} & =\left[\mathbf{y}(-M)^{T}, \ldots, \mathbf{y}(M)^{T}\right]^{T} \\
& =\sum_{n=0}^{L-1} \sum_{l=0}^{L-1} \alpha(n) \alpha(l) \mathbf{R}(l-n) \mathbf{A} \mathbf{b}+\mathbf{z}
\end{aligned}
$$

where $\mathbf{y}=\left\{y_{1}, \ldots, y_{K}\right\}^{T}$.

Making $\mathbf{R}=\sum_{n=0}^{L-1} \sum_{l=0}^{L-1} \alpha(n) \alpha(l) \mathbf{R}(l-n)$ the matrix $\mathbf{R}$ can, now, be interpreted as the cross-correlation matrix for an equivalent synchronous AWGN problem where the whole transmitted sequence is considered to result from $(2 M+1) \times K$ users, during one transmission interval of duration $T_{c}=(2 M+1) \times T_{b}$. The matrix $\mathbf{R}$ is not symmetric anymore. The vector of decision statistics becomes now $\mathbf{y}=\mathbf{R} \mathbf{T} \mathbf{A} \mathbf{b}+\mathbf{z}$. Now, a multiuser detection problem in multipath chan nels is reduced to $\mathrm{K}$ decoupled single user detection problems. 
4 Mário Marques da Silva1, Américo Correia2

\section{System Model for theSelective Transmit Diversity}

The STD scheme has a low rate $(750 \mathrm{~Hz}-3 \mathrm{kHz})$ feedback link from the receiver (MS) telling the transmitter (BS) which antenna should be used in transmission. There is a common or dedicated pilot sequence, which is transmitted. Different antennas with specific pilot patterns/codes enable antenna selection. Then, the transmitter has a link quality inform ation about the $M$ (number of antennas) links and it transmits a single symbol stream over the best antenna. The receiver is supposed to re-acquire the carrier phase after every switch between antennas. The Antenna Switch has the capability to switch every slot duration, i.e., $0.667 \mathrm{~ms}$ (rate 1.5 $\mathrm{kHz}$ ). After using STD from $M$ antennas over the $L$-paths, each with equal average energy, then we have changed the probability density function for each link. The BER analytical expression is described in [5]. The decision for the antenna selection of the STD combined with the RAKE receiver (maximize multipath diversity) is done in order to select the antenna $i \in[1, \ldots, M]$ which maximizes the multipath diversity, i.e., maximizes the sum of the square of the absolute values of the paths gains, which corresponds to maximize $\max \left(\sum_{l=1}^{L}\left|h_{i l}\right|^{2}\right)$.

\section{Results and discussion}

All the simulation results presented in this section considered the QPSK modulation in a frequency selective Rayleigh fading channel, namely the Vehicular A prop agation model of 3GPP. This propagation models was selected to evaluate the effect of IPI in the performance of the proposed PC scheme. Thus, the Vehicular A propagation model presents a high number of paths, whose average power profile is modelled

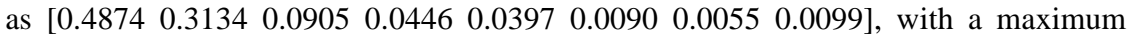
delay spread correspondent to seven chip periods.

The Walsh-Hadamard spreading sequences were considered in the simulation, with spreading factor 16 and 64. Positions of interfering MSs were defined randomly using a uniform distribution inside a cell, as well as the correspondent CIRs for different users. If we consider all users co-located with the reference user, i.e., same CIRs for all users, performances are better than those presented in this paper. As can be seen in Fig. 1 for $\mathrm{SF}=64$ and 15 users, an increase in the order of diversity to $2 L$ (where $L$ corresponds to the number of multipaths) with the use of the STD, corresponds to an improvement of performance. However, due to the presence of MAI, we can see that the improvement of performance with the increase of $E_{b} / N_{0}$ is low. This was the main motivation to add the proposed PC scheme to the STD, whose results correspond to a gain over the STD alone. The use of the PC corresponds to an appreciated performance improvement over the "Only RAKE", except for low values of $E_{b} / N_{0}$, where the noise enhancement generated by the decorrelator type precoding reaches a high value. The PC is able to partly "clean" the MAI which was generated due to the presence of the multipath environment. Additionally, the best performance is achieved with the combined scheme composed of STD and PC. 


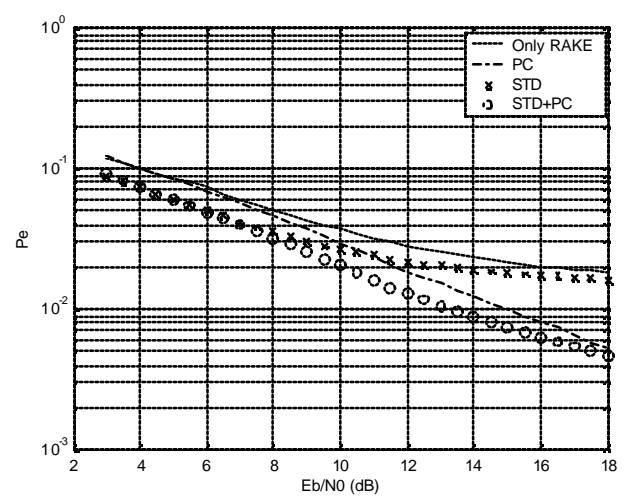

Fig. 1. BER performance for 15 users, $S F=64$

The results of Fig. 2a) are similar to the previous one, except that we now consider $\mathrm{SF}=16$. Similar results were obtained, with the exception that, in this case, the performance obtained with STD+PC only outperforms the STD for values of $E_{b} / N_{o}$ below $11 \mathrm{~dB}$. There are two steps of performance: the first corresponds to the system without any kind of PC (with and without STD), and hence, with a high level of MAI; the second corresponds to the system in which the PC was used to remove part of the MAI, which originates a step in the performance.
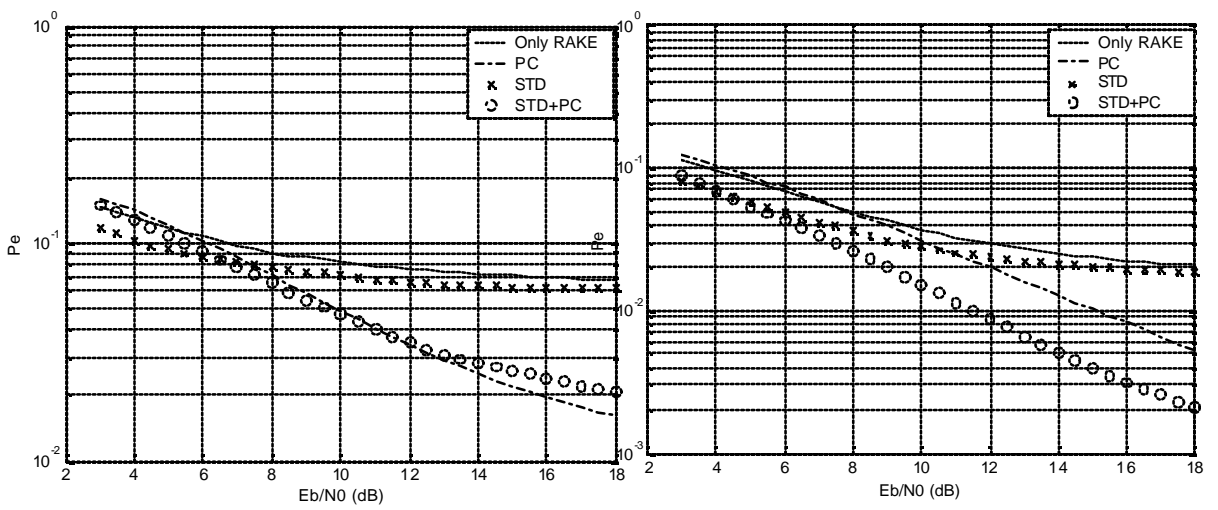

Fig. 2. BER performance for $\mathrm{SF}=16$, with a) 15 users and b) 4 users

Due to lower level of MAI in Fig. 2b), the gain obtained with the PC (relating to "Only RAKE") is lower than that of Fig. 2a). The performance improvement of the STD+PC relating to STD is much higher than the performance improvement of the PC relating to the "Only RAKE". This confirms that the system considering the STD+PC is able to achieve a perform ance with very low level of bit errors. With four users, the gain obtained with STD+PC relating to "Only RAKE" is much higher than with 15 users. This happens once the system is "cleaner" of MAI and, hence, the STD is able to reach a better performance. 
6 Mário Marques da Silva1, Américo Correia2

\section{Conclusions}

In this paper we have studied the combination of two specific schemes, namely the precoding scheme with the selective transmit diversity for the downlink, through the Vehicular A propagation models of 3GPP. When the level of MAI was more intense we observed that, even with the use of the STD, the performance obtained was limited. This was the main motivation to add the PC to the STD scheme. It was shown that the use of the PC combined with the STD tends to reach a performance improvement over the corresponding schemes isolated. This tends to happen because the PC "cleans" the MAI and, hence, the diversity provided by the use of the STD is able to achieve better results. This confirms that the combination of STD and the PC fit very well for the downlink 3 GPP.

Our work considered the vehicular A propagation model which presents higher number/level of multipath diversity, and consequently, originates higher level of MAI. However, once most of propagation models of 3GPP present an order/level of multipath diversity similar to the Vehicular A propagation model, we should conclude that the combination of STD with PC tends to achieve better results in several propagation environments.

\section{Acknowledges}

This work has been partially funded by the IST -2003-507607 EU $\underline{B}$ roadcasting and

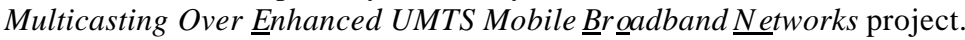

\section{References}

1. B. R. Vojcic, W. M. Jang, "Transmitter Precoding in Synchronous Multiuser Communications", IEEE Transactions on Communications, Vol. 46, n. ${ }^{\circ} 10$, October 1998, pp. 1346-1355.

2. J. Choi, "Interference Mitigation Using Transmitter Filters in CDMA Sy stems", IEEE Transactions on Vehicular Technology, nº-Vol.51, July 2002, pp. 657666.

3. M. M. Silva, A. Correia, "Interference Cancellation with combined Predistortion filtering and Transmit Diversity", $14^{\text {th }}$ IEEE International Symposium on Personal Indoor and Mobile Radio Communications, Beijing, China, 710 September 2003.

4. M. M. Silva, A. Correia, "Joint MultiUser Detection and Intersymbol Interfe rence Cancellation for WCDMA Satellite UMTS", Internation Jounal of Satellite Communications and Networking - Special Issue on Interference Cancellation John Willey and Sons, Ltd, n. ${ }^{\circ}$ 21, 2003, pp. 93-117.

5. A. Correia, "Optimized Complex Constellations for Transmission Diversity", Wireless Personal Communications 20, Journal of Kluwer Academic Pub, Mar. 2002, pp. $267-284$. 